\title{
Dynamic Reconfiguration under RTEMS for Fault Mitigation and Functional Adaptation in SRAM-based SoPCs for Space Systems
}

\author{
Arturo Pérez, Leonardo Suriano, Andrés Otero, Eduardo de la Torre
}

I. INTRODUCTION

On-board computing systems used in space applications must comply with the so-called SWaP (Size, Weight and Power) restrictions, whilst ensuring very high levels of reliability. The foremost threat to that end is the presence of radiation in the environment that, in the form of high-energy particles [1], can induce faults in the microelectronic devices used in the spacecraft. The most effective way to protect electronic systems operating under these conditions is to use devices hardened by technology, such as the PowerPC 603e/sup or the RAD750 single board computers, shown in [2] and [3] respectively. Hardened devices can ensure high reliability levels, although its performance is generally below than what commercial-of-the-shell (COTS) devices can offer, incurring also in much higher implementation costs.

As complexity and lifetime of space systems rise, COTS FPGAs based on static random access memories (SRAM) are becoming an increasingly attractive alternative to replace hardened devices in spaceborne high performance processing systems [4]. On top of offering a high computational power with a relatively low power consumption, unequalled flexibility enabled by the reconfiguration capability of SRAM FPGAs

opens the door to the modification of the functionality of the whole device during its lifetime according to the different scenarios a spacecraft may encounter during a mission, as envisaged in [5]. An even greater flexibility may be achieved by using the dynamic and partial reconfiguration (DPR) mechanism offered by some FPGA devices. DPR consists in the modification of a Reconfigurable Partition of the FPGA while the rest of the device is powered on and remains working [6]. By exploiting this technique, it is possible to reutilize the same hardware resources to run non-simultaneous functionalities, saving logical resources and power.
Beside these benefits, the use of SRAM-based FPGAs in such a harsh environment poses a twofold challenge: both data memory and configuration memory can be corrupted by the occurrence of faults. Modifications in the data memory might cause the production of temporally or permanently erroneous output values, something that can be mitigated by applying well-known techniques, such as Triple Modular Redundancy (TMR). In case the configuration memory is affected, the functionality performed by the device will differ from the original one, generating unexpected behaviors.

In turn, faults in the configuration memory can be temporal or permanent [7]. On the one hand, temporal faults do not have a destructive effect and therefore they can be recovered by different scrubbing and readback techniques. The simplest mitigation technique is blind scrubbing, which consists in periodically writing in the configuration memory using data from a golden copy. This way, possible Single Event Upset (SEUs) and Multiple Event Upset (MEUs) present in the configuration memory are overwritten with the original configuration file. Another strategy is readback scrubbing, which is based on the principles of detection and correction. This strategy consists in checking the configuration memory searching for upsets. Only when at least one upset is detected, the correction mechanism is triggered to fix the error(s). On the other hand, permanent faults in SRAM-based FPGAS are mainly due to long time effects, which are referred to as the Total Ionizing Dose (TID). Under these hard faults, rewriting the content of the configuration memory is no longer a useful solution. However, dynamic reconfiguration capability of the FPGAs can be exploited to re-allocate Reconfigurable Modules 
(RM) in alternative Reconfigurable Partitions when part of the FPGA is damaged.

The use of DPR in the space environment might therefore have a twofold purpose: to share resources among different functionalities and the relocation of modules from damaged to fault-free regions. In addition, the use of scrubbing techniques also involves accessing the configuration memory, so it is necessary to include arbitration mechanisms to deal with the potential conflicts that may appear when accessing this memory. Moreover, the rate at which fault detection and mitigation techniques must be applied will be conditioned by the frequency of the emergence of faults caused by radiation. Therefore, the system must be also able to trigger the fault recovery mechanism with a variable period that must be adaptively adjusted according to the error detection rate [1].

These arbitration and timing requirements may be fulfilled by the use of a Real Time Operating System (RTOS) that provides a reliable software environment to build applications with a deterministic performance, while increasing the portability of the application codes, fostering code reuse and therefore saving time and reducing costs for the programmers.

In this context, a complete integration of a read-back scrubbing strategy (used to detect errors and damaged areas of the FPGA) and DPR is proposed in this paper. DPR is used both to repair eventually corrupted frames of the configuration memory or by simply changing the functionalities of the whole device. Both mechanisms have been integrated on a hybrid Xilinx Zynq 7000 family device, which is a heterogeneous SoPCs (System on Programmable Chip) that combines a hardwired ARM CortexA9 dual-core processor with a reconfigurable FPGA, in the same chip. A real time operating system has been also ported to the ARM processors on the Zynq to control the access to the reconfiguration memory as well as to deal with the timing deadlines needed for each of those mechanisms. In particular, the Real-Time Executive for Multiprocessor Systems (RTEMS), which is an open source Real Time Operating System widely used in medical, industrial and aerospace applications, has been selected. Finally, a reconfigurable image processing pipeline, that includes functionalities used in autonomous visual navigation applications, has been also integrated in the system, as a proof of concept of the proposed platform.

The rest of this paper is organized as follows: first, a description of the state of the art is introduced, focusing on the usage of SRAM-based FPGAs and real time operating systems in space applications. Then, the proposed platform is shown, together with the design methodology envisaged to develop a system on top of this platform. Finally, implementation results and future work are presented.

\section{STATE OF THE ART AND BACKGROUND}

Before describing the platform and the proposed approach for the integration of the Scrubber and the Dynamic Reconfiguration mechanism within the services offered by RTEMS, previous works and background information reporting the use of these technologies in space systems are analyzed in this section.

\section{A. SRAM based Heterogeneous SoCs in Space Applications}

The industrial and academic interest in developing platforms for space on-board computing based on heterogeneous SRAMbased devices, such as those belonging to the Zynq family, is clear from the analysis in the State-of-the-Art. A good example is the APEX-SoC, proposed by Xabier Iturbe et al. in the NASA Jet Propulsion Laboratory (JPL) [8]. APEX-Soc is a platform to control science instruments proposed for future NASA missions. Authors use the Compositional InfraRed Imaging Spectrometer (CIRIS), an instrument that is planned to be used in future NASA moon missions, as a use-case scenario to demonstrate that the whole system, including data processing, control and interfaces, can be implemented on the single chip.

A similar approach was followed by the CHREC Space Processor (CSP) where University of Florida researchers, together with the NASA Goddard SpaceCube team, developed a hybrid space computer exploiting both radiation-hardened and Commercial-Off-the-Shell (COTS) devices making use of different fault tolerant computing strategies [9].

\section{B. Dynamic and Partial Reconfiguration in the Space Environment}

From the previous analysis, it is clear that in order to exploit the advantages of reconfigurable devices in the space environment, it is absolutely necessary to develop methodologies and Fault-Mitigation-Techniques to make systems more robust and reliable. These techniques were deeply studied and applied by Luca Sterpone et al. in [10], where a novel fault tolerant and runtime reconfigurable platform for satellite payload is proposed. In particular, blind and readback scrubbing together with TMRs strategy were used to develop a new algorithm for the analysis of critical radiation effects in order to obtain an effective estimation of the radiation impact and enabling the tuning of the component mapping. In turn, Bolchini and Sandionigi proposed in [7] a classification algorithm to discriminate between recoverable and not recoverable faults in SRAM-based FPGAs with the final aim of exploiting these powerful devices even in space applications.

Regarding the reconfigurable image processing pipeline proposed in this paper as a proof of concept, a similar approach can be found in [11]. From this work, it is clear that the needing of hardware accelerator to meet real-time constraint cannot be under-evaluated. In fact, Khalifat propose the use of Dynamic Partial Reconfiguration to exploit the FPGA computational power, allowing time sharing of the reconfigurable resources between different video applications, thus increasing the area efficiency.

\section{Real Time Operating Systems for Space Applications}

RTEMS is an operating system widely used in space applications [12], making it a good candidate to be implemented as the system support for the reconfigurable processor.

RTEMS has been designed having in mind the portability through different hardware platforms by using a multi-layered approach as illustrated in Figure 1. The source code is 
organized by CPU families with a variety of processors for each family. RTEMS takes advantage of similarities between processor families by reusing software, allowing the enabling or not of each specific system feature [13]. For each processor, a set of Board Support Packages (BSP) is available, where the board initialization routines as well as specifics drivers can be found.

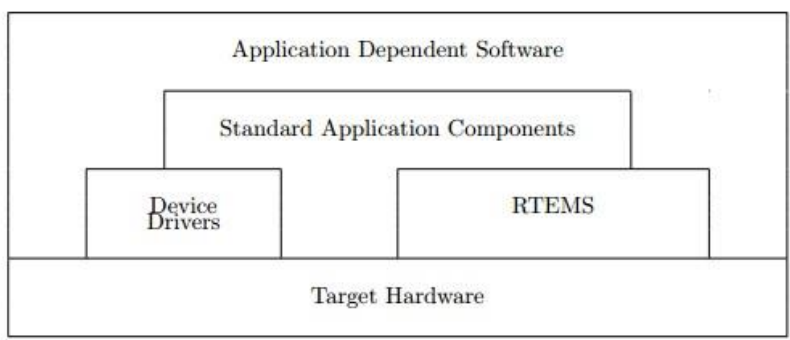

Figure 1. RTEMS multilayered structure from [12]

The interface, exposed to the application, contains a set of directives, called resource managers, grouped by the functionality that they manage. They offer services such as the scheduler, task support, clock management or errors managers, among others, used to build real-time applications. All of them are based on a set of CPU independent functions that compounds the RTEMS kernel, also called the SuperCore or the executive [12]. This organization is sketched in Figure 2. It is also important to mention that RTEMS supports open standard APIs such as POSIX [14].

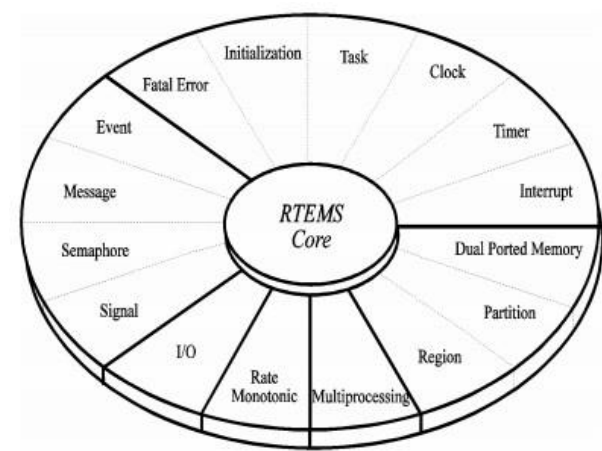

Figure 2. RTEMS architecture [13]

From a technical point of view, the most important features of RTEMS are [13]:

- $\quad$ Flat memory model: the correspondence between physical and virtual memory is one to one.

- $\quad$ Single-process, multiple-threads.

- It supports Symmetric Multiprocessing (SMP) execution.

- Event-driven, priority-based, preemptive scheduling.

- Optional rate monotonic scheduling.

- Inter-task communication and synchronization.

- Priority inheritance.

- Responsive interrupt management.

- Dynamic memory allocation.

- High level of user configurability.
For the case of the reconfigurable processor, a series of scrubbing techniques, such as those mentioned in section A, combined with other periodic tasks like self-monitoring for self-awareness, need to operate in combination with mission tasks that require strong predictability characteristics. For this reason, the RTEMS OS was selected.

\section{RECONFIGURABLE PROCESSOR ARCHITECTURE}

In this section, a description of the architecture and the functionality of the proposed reconfigurable processor is provided.

Main features of the platform are the dynamic and partial reconfiguration and the scrubbing mechanism that have been integrated as system support tasks on top of the RTEMS operating system. Scrubbing and DPR can be considered as critical system support functions because they are intended to increase the flexibility and the survival time of the platform.

The Zynq-7000 SoC architecture, selected to be used in this work, is split into two parts. On one side it is the Processing System (PS) where two ARM Cortex-A9 processors are implemented in fixed logic. On the other side it is the Programmable Logic (PL) part, where different soft IPs can be implemented using reconfigurable resources.

The block design, corresponding to the proposed system, can be represented as shown in Figure 3. The Application Processing Unit (APU) corresponds to the ARM A9 processors where the RTEMS application runs. In turn, the highlighted zone inside the PL corresponds to the Reconfigurable Region and it contains the amount of resources needed to implement any of the image filters provided. The other blocks presented in the static part of the PL are necessary in order to have a serviceable device. All the operations related with the PL configuration must be done by means of reading and writing operations on the registers of the configuration module. This module is embedded in the PL and can be accessed from the PS using the Device Configuration interface (DevC). The DevC offers the possibility of initializing and configuring the PL from the software program running in the PS, by using the Processor Configuration Access Port (PCAP) Bridge. The PCAP Bridge is a gateway which interfaces, on one side, with the PS Advanced eXtensible Interface (AXI) Interconnect and, on the other side, with the configuration module through the PCAP interface. Figure 4 gives an overview of this subsystem.

To reconfigure the FPGA is required to introduce certain commands through the PCAP, together with the configuration data (bitstream) corresponding to the module to be loaded in the reconfigurable region. Similarly, to read the configuration memory, first the commands requesting the operation are sent, and then, configuration can be read. Further information on the configuration procedure is offered in the manufacturer's design guides [15]. Required data transactions are performed by a DMA controller embedded in the $\mathrm{DevC}$, which is 
controlled by means of registers and it is a master of the AXI PS interconnect.

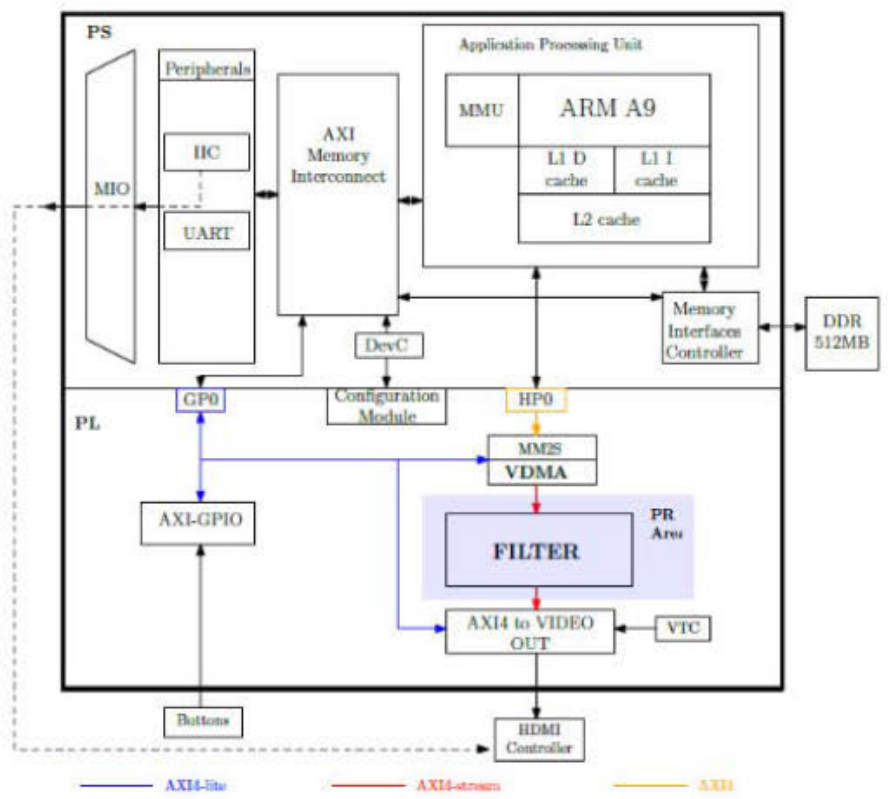

Figure 3. System Block Design

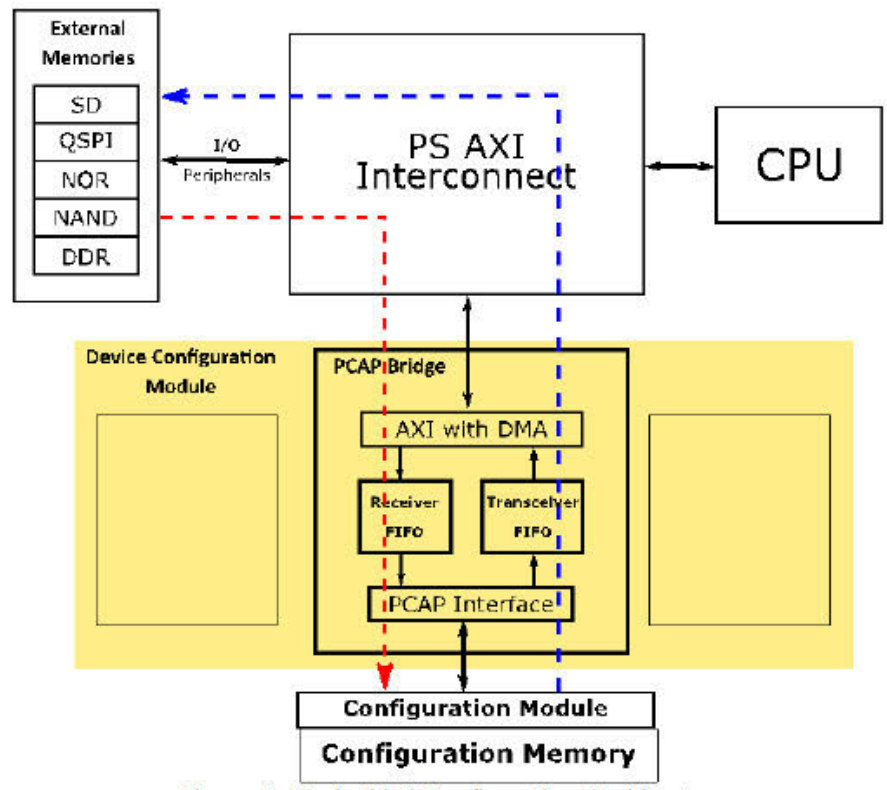

Figure 4. Embedded Configuration Architecture

The application running under the RTEMS support is in charge of managing the whole system where Rate monotonic manager is used to implement a deterministic execution of the support functions. The execution flow of this application can be seen in Fig. 5. It performs periodically a basic readback scrubber comparing the whole configuration memory with a golden copy and in case any error is detected, it reconfigures the FPGA to fix it. In order to identify the bits of the configuration memory carrying information regarding the functionality of the FPGA, masks files must be used by the scrubber. To mimic an external event triggering a DPR demand external buttons available in the development board were used. When any of them is pushed, the dynamic region of the PL is reconfigured with a different filter. The scrubber and DPR functions use the same physical resource of the system (the DevC to access the configuration module), so they must be synchronized to avoid conflicts. To this aim, a semaphore provided by RTEMS was implemented, showing the benefits of using a real-time operating system for the control of the system critical tasks.

A reconfigurable image processing pipeline has been included in the system as a potential compute demanding function to be used in missions using visual guidance algorithms [16]. It aims to be a proof of concept of the system. It consists of three representative alternative accelerators:

- Sobel-Felman Operator, a very popular image processing algorithm used meanly for Edge Detection. This is also one of the fundamental brick used by other computationally intensive algorithm.

- Harris Detection Corner, very resource demanding (it works with floating point data), used for image feature detecting.

- Bypass filter, used just to check if the image was altered by any of the steps of the video pipeline.

These algorithms are typical core functions of many autonomous or smart vision systems where, thanks to the information extracted by analyzing camera frames, the system can interact with the surrounding world. The HW accelerators were created using HLS techniques, as it will be described in the next section.

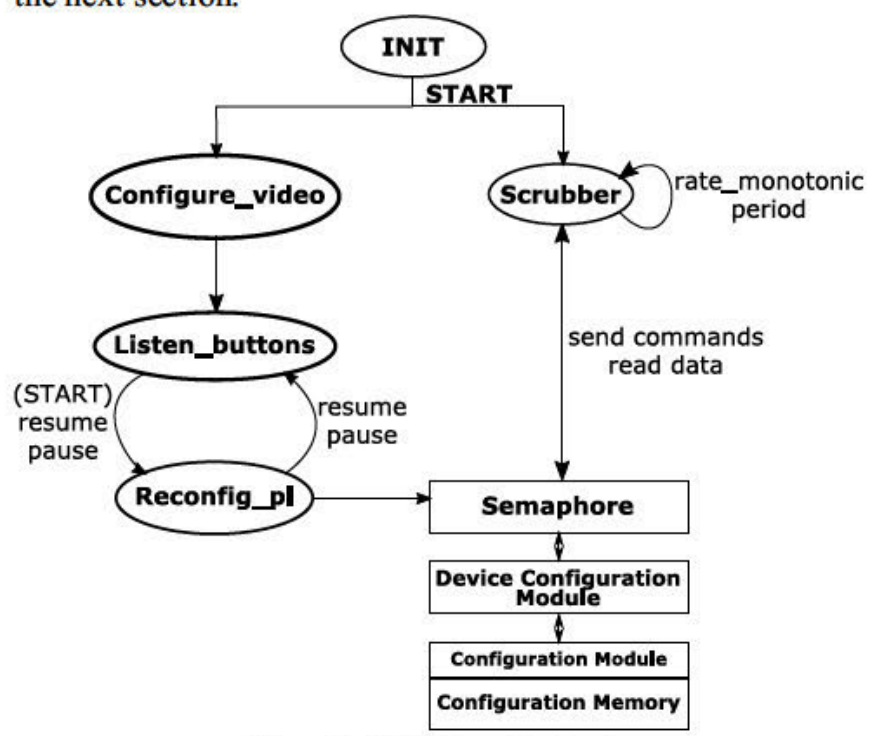

Figure 5. RTEMS application flow

Four different configuration bitstream files were generated for the system: one for the static part and three files corresponding to each HW accelerator to be used in the reconfigurable region. Partial bitstreams with the configuration information 
of the HW accelerators are stored in the external DDR memory of the device, being available to be used by the application running under RTEMS when required.

\section{RECONFIGURABLE ACCELERATORS DESIGN FLOW}

In this section, the main steps followed for the design of the reconfigurable image processing accelerators on top of the proposed platform are described. The overall flow is shown in Figure 6 and it uses the commercial tools developed by Xilinx.

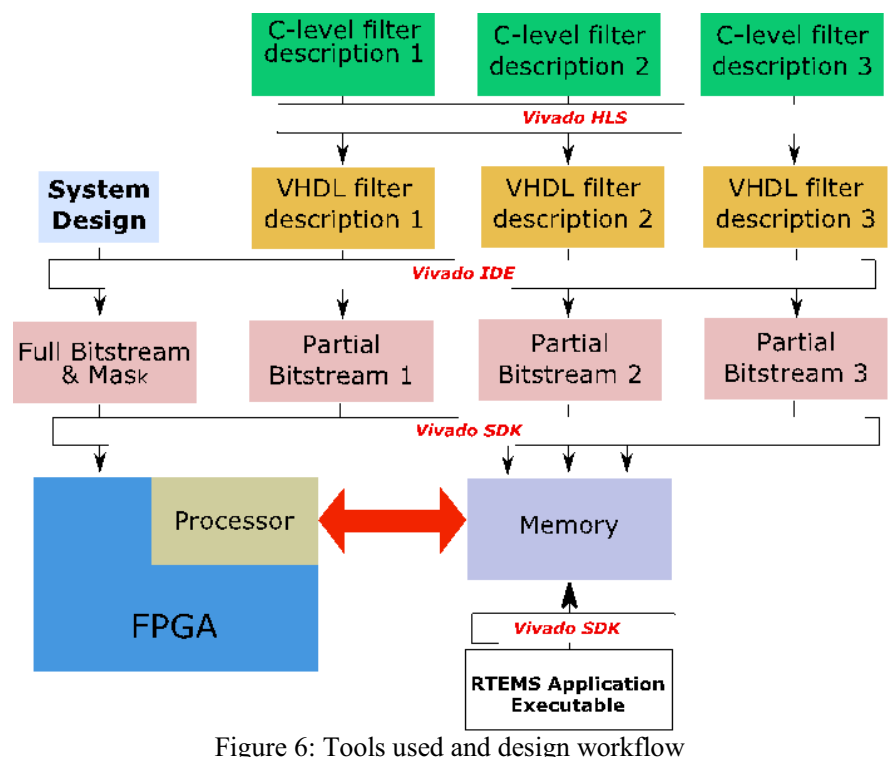

\section{A. Implementation of the Image Processing Accelerators}

Reconfigurable image processing filters have been created using HLS tools. These tools allow describing the algorithms to be implemented in hardware using $\mathrm{C} / \mathrm{C}++/$ SystemC languages, rising the design abstraction level though hiding the low level details of the design, in comparison with the use of RTL descriptions. In particular, the tool used in this work was Vivado HLS. An important role in the design of the accelerators was also played by the Open Source Computer Vision Library (OpenCV [17]) and by the synthesizable Vivado HLS Video Libraries [18]. Once the algorithm is evaluated, the tool translates the code to an HDL (VHDL or Verilog) having the possibility of incorporating it in a more complex RTL-design. It is possible to drive the translationprocedure by adding directives and constraints in order to, for example:

- $\quad$ specify the nature of the protocol of the input/output ports,

- choose the level of pipeline and the level of parallelism of the algorithm,

- select the specific resources to use,

- condition the scheduling of the functions/loops,

- decide how to access to the memory.
For this particular case, it was chosen a streaming interface in order to maximize the throughput of the system: in this way, also, it results natural the using of pipeline strategy to speed up the for-loops inside the image processing function.

The last part regarding the building of a working system able to processes images is the generation of the bitstreams to configure the PL part of the FPGA as described in the next section.

\section{B. Generation of Dynamically Reconfigurable Bitstreams:}

In order to obtain the bitstreams and the masks files, the commercial Partial Reconfiguration workflow developed by Xilinx [19] was followed. This means, among other things:

- To synthetize a design using a bottom-up approach, as described in [20];

- To identify the physical area upon the FPGA (Configuration Region) where to create a valid floorplan for a partial reconfiguration design;

- To create the all configurations with the common static section;

- $\quad$ To implement (optimization, placement and routing) the different configurations;

- To save the bitstreams that will be managed by the Operating System chosen.

The generated bitstreams could be used to repair any part of the design affected by upsets or to modify, at runtime, the device functionality as it described in section III. Moreover, if the application needs different features, other filters can be run by reusing the same silicon resources, without requiring a larger FPGA. In fact, it is impossible to fit the three filters together, on the device chosen, because of timing routing errors.

\section{RTEMS ON ARM A9 ZYNQ PROSCESORS}

RTEMS is distributed with a set of tools easing the process of building applications, called the RTEMS Source Builder (RSB). When a new design is done everything has to be built from the sources allowing the designer to control each step of the process. For the case of the ARM A9 processors included within the Zynq device, where the reconfigurable processor was implemented, the steps shown below were followed:

- The RTEMS Source Builder (RSB) was built for the ARM processor family and for RTEMS version 4.12 using GNU autotools.

- The sources of the RTEMS project were built for the development board (Zedboard) Board Support Package (BSP) using RSB and GNU autotools. The generated libraries were installed on the host PC to be used for building RTEMS applications.

- Drivers were generated to manage peripherals present in the PL or PS. 
Once the porting was finished, the code of an RTEMS application was created using the drivers and the RTEMS BSP libraries. The application starts configuring the Memory Management Unit (MMU) as is required. The application executable was created building the application source code using the RSB and standard GNU tools. All these steps are summarized in Figure 7:

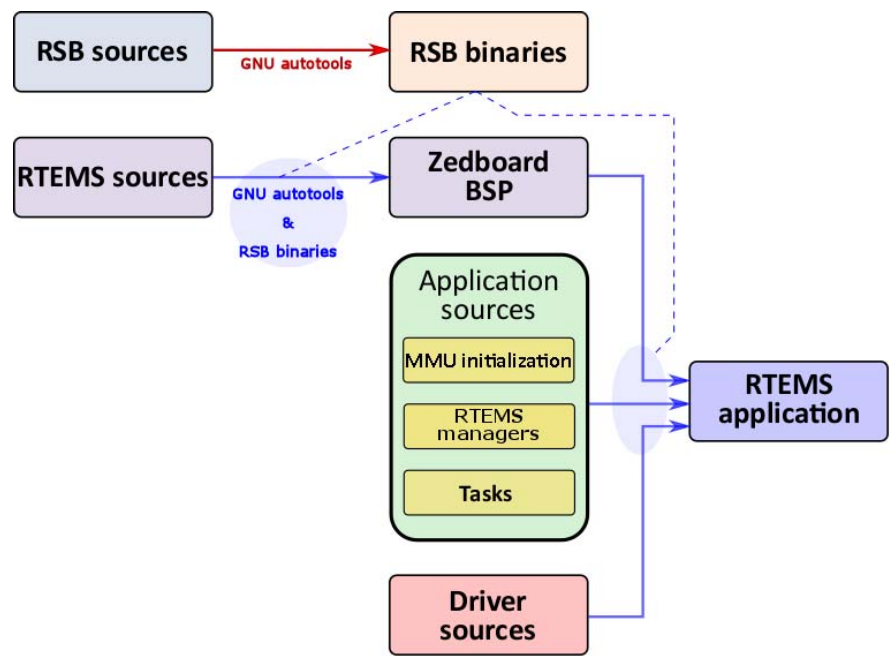

Figure 7 . RTEMS porting flowgram

RTEMS uses a flat memory model, instead of using virtual memory, as is usually the case of non-critical operating systems. Besides this, the MMU in the ARM A9 processors must be configured to map the memory regions used by each application. This way, if an application tries to access to a memory region not mapped by the MMU, an abort exception occurs and the application is halted. In the case of RTEMS, as the application is viewed as a single process, all the system is aborted. This is a critical issue when new devices are added to the PL because they are controlled by using memory mapped protocols. To overcome this situation, the MMU is configured in the initialization routines of the RTEMS application, assigning pages to the memory mapped peripherals with device attributes.

\section{IMPLEMENTATION RESULTS}

This section exposes measures of the most representative parameters of the system described in section III, including the memory footprint required by the system and the FPGA resources used by the design.

\section{A. System Implementation}

The layout of the design implemented in the FPGA can be seen in Figure 8, where it is possible to notice the position of the static part of the design (orange) and the dynamic part (blue). Reconfigurable area can be reused to allocate any of the implemented filters for the image processing pipeline.

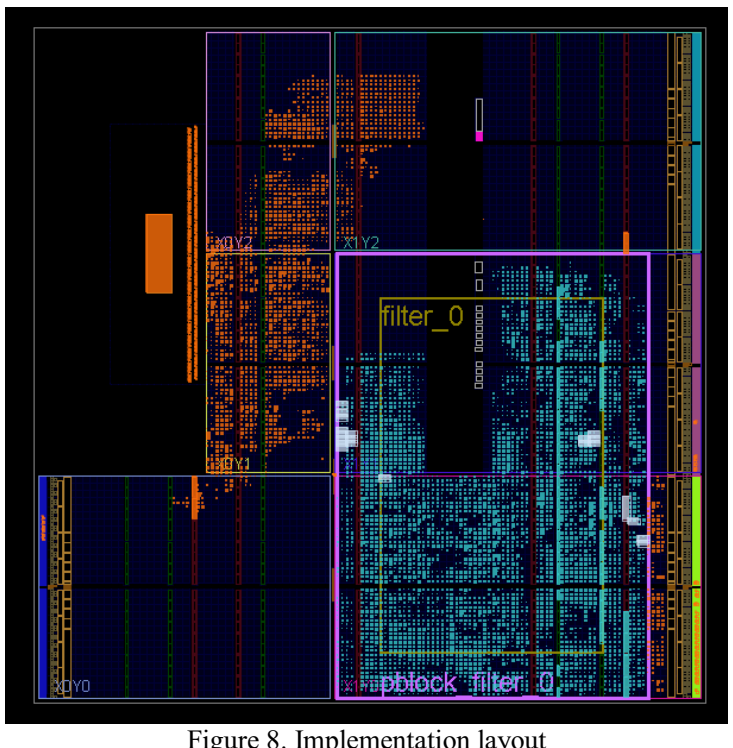

Figure 8. Implementation layout

The device chosen for the proof of concept is the Zynq 7020 from the Zynq 7000 family.

\section{B. Performance metrics}

Measuring the performance of some functions has been carried out by counting the clock cycles taken for each task from the processor registers.

\section{1) Dynamic Partial Reconfiguration}

Table 1 shows the time needed to load a new partial bitstream corresponding to the reconfigurable region (5800 slices, 80 DSP48s, 120 RAMB18 and 60 RAMB36) into the configuration memory through the PCAP port working at a frequency of $200 \mathrm{MHz}$.

Table 1: DPR metrics.

\begin{tabular}{|c|c|c|c|}
\hline & Time (us) & $\begin{array}{c}\text { Throughput } \\
(\mathrm{MB} / \mathrm{s})\end{array}$ & $\begin{array}{c}\text { Data size } \\
(\mathrm{MB})\end{array}$ \\
\hline DPR & 9999.210 & 130.189 & 1.302 \\
\hline
\end{tabular}

\section{2) Scrubber}

For the Scrubber function the time needed to send the readback commands and to read the configuration memory can be seen in Table 2. The PCAP clock was configured to $100 \mathrm{MHz}$. It is important to remark that different frequencies were used for reconfiguring and for reading the PL: it has been empirically checked that the data results degraded when reading the configuration memory at frequencies higher than $100 \mathrm{MHz}$. Instead, just reconfiguring the FPGA with higher frequencies doesn't affect the correctness of the operations. 
Table 2: Readback metrics

\begin{tabular}{|c|c|c|c|}
\hline $\begin{array}{c}\text { Data } \\
\text { Transaction }\end{array}$ & Time (us) & $\begin{array}{c}\text { Throughput } \\
(\mathrm{MB} / \mathrm{s})\end{array}$ & Data size \\
\hline $\begin{array}{c}\text { Readback } \\
\text { commands }\end{array}$ & 2.634 & 84.5 & $220 \mathrm{~B}$ \\
\hline $\begin{array}{c}\text { Read } \\
\text { configuration } \\
\text { memory data }\end{array}$ & 20217.188 & 199.98 & $4.043 \mathrm{MB}$ \\
\hline
\end{tabular}

The time needed to check the data can be seen in Table 3. For this task, two tests have been done, using cache memories or not.

Table 3: Checking bitstreams time.

\begin{tabular}{|c|c|}
\hline Operation mode & Time (us) \\
\hline $\begin{array}{c}\text { Without using cache } \\
\text { memories }\end{array}$ & 207651.844 \\
\hline Using cache memories & 20444.158 \\
\hline
\end{tabular}

These tests show that the time needed to perform the same operation, using or not cache memories, varies in the order of ten times less for a cache-less solution.

\section{Resource usage}

The amount of resources needed by the design is shown below. In Table 4, the resources needed by the static part and by each design of the dynamic part can be seen.

Table 4: PL resource utilization

\begin{tabular}{|c|c|c|c|c|}
\hline Resource & $\begin{array}{c}\text { Static } \\
\text { Part }\end{array}$ & $\begin{array}{c}\text { Sobel } \\
\text { Filter }\end{array}$ & $\begin{array}{c}\text { Harris } \\
\text { Filter }\end{array}$ & $\begin{array}{c}\text { Full } \\
\text { Design }\end{array}$ \\
\hline LUT & 3682 & 1067 & 10105 & 14854 \\
\hline FF & 4754 & 1297 & 11966 & 18017 \\
\hline BRAM & 4 & 3 & 18 & 25 \\
\hline DSP & 47 & 0 & 47 & 94 \\
\hline LUTRAM & 429 & 0 & 429 & 858 \\
\hline
\end{tabular}

The upper table shows the big amount of resources by the PL full design (which represents the sum of the resources needed if the design would have been implemented without using the DPR). Thanks to the use of this technique is possible, in fact, to reduce the amount of total resources by sharing logic between filters. In this way, it is possible to reduce the power consumption of the chip and increase, at the same time, the flexibility of the FPGA.

\section{Memory footprint}

The system memory usage is reported in Table 5, for the implemented solution.
Table 5: Memory footprint

\begin{tabular}{|c|c|c|}
\hline File & $\begin{array}{c}\text { Memory } \\
\text { utilization by one } \\
\text { file (MB) }\end{array}$ & $\begin{array}{c}\text { Total memory } \\
\text { utilization (MB) }\end{array}$ \\
\hline Executable & 4.3 & 4.3 \\
\hline Partial bitstream & 1.3 & 3.9 \\
\hline Mask Files & 4.45 & 16.2 \\
\hline $\begin{array}{c}\text { Original } \\
\text { bitstream }\end{array}$ & 4.45 & 16.2 \\
\hline
\end{tabular}

These results show that a big amount of memory must be reserved for the files used to check the configuration memory during the scrubbing process. Differently, the memory needed for the RTEMS application is small. At any rule, given the cost of hardened memories for space applications, bitstream and mask compression will be an interesting future line of research as demonstrated in [21].

\section{CONCLUSION AND FUTURE WORKS}

This paper validates the integration of a scrubbing and a dynamic reconfiguration mechanism under the support of RTEMS, as the core of a reconfigurable processor for space applications. This way we demonstrate how the RTOS can manage the dynamic reconfiguration for both changing the functionality of the hardware (increasing so the flexibility and the on-board computing power) and fixing bugs detected by the readback of the configuration memory, while solving the conflicts with the scrubber when accessing the device configuration memory. Hence, this approach increases the reliability and flexibility of the global system.

Further improvements on this platform are being carried out, affecting to the performance of the reconfiguration process (including extra features such as reallocation of partial bitstreams), extending the RTEMS drivers for other resources available in the Zynq device or enhancing the scrubbing technique, among others. Validating the correct operation of the fault mitigation techniques developed in this work, and the more complex ones that are under development, will be carried out by injecting faults in the HW design, using the Xilinx's proprietary SEM IP [22].

\section{ACKNOWLEDGEMENTS}

This work has been partially supported by the Enable-S3 project that has received funding from the ECSEL Joint Undertaking under grant agreement No 692455. This joint undertaking receives support from the European Union's HORIZON 2020 research and innovation programme and Austria, Denmark, Germany, Finland, Czech Republic, Italy, Spain, Portugal, Poland, Ireland, Belgium, France, Netherlands, United Kingdom, Slovakia and Norway. This work has also been supported by the Spanish R\&D State Plan under grant PCIN-2015-254. Leonardo Suriano holds a predoctoral contract under RR01/2015 (Programa Propio) by Universidad Politécnica de Madrid. 


\section{REFERENCES}

[1] J. L. Barth, C. S. Dyer and E. G. Stassinopoulos, "Space, atmospheric, and terrestrial radiation environments," in IEEE Transactions on Nuclear Science, vol. 50, no. 3, pp. 466 482, June 2003.

[2] G. R. Brown, "Radiation hardened PowerPC 603eTM based single board computer," 20th DASC. 20th Digital Avionics Systems Conference (Cat. No.01CH37219), Daytona Beach, FL, 2001, pp. 8C1/1 8C1/12 vol.2.

[3] Berger, R.W. et. al., "RAD750 SpaceWireEnabled Flight Computer for Lunar Reconnaissance Orbiter," Proc. of International SpaceWire Conference, Dundee, Scotland, Sep. 2007.

[4] R. Ferguson and R. Tate, "Use of Field Programmable Gate Array Technology in Future Space Avionics," Proc. 24th Digital Avionics Systems Conf. (DASC '05), vol. 2, p. 11, Oct /Nov. 2005.

[5] N. Steiner and P. Athanas, "Hardware autonomy and space systems," 2009 IEEE Aerospace conference, Big Sky, MT, 2009, pp. 113.

[6] A. Otero, E. de la Torre and T. Riesgo, "Dreams: A tool for the design of dynamically reconfigurable embedded and modular systems," 2012 International Conference on Reconfigurable Computing and FPGAs, Cancun, 2012, pp. 18.

[7] C. Bolchini and C. Sandionigi, "Fault Classification for SRAM Based FPGAs in the Space Environment for Fault Mitigation," in IEEE Embedded Systems Letters, vol. 2, no. 4, pp. 107 110, Dec. 2010.

[8] X. Iturbe et al., "Towards a generic and adaptive System on Chip controller for space exploration instrumentation", in the 2015 NASA/ESA Conference on Adaptive Hardware and Systems (AHS), Montreal, QC, 2015, pp. 18.

[9] D. Rudolph, C. Wilson, J. Stewart, P. Gauvin, A. George, H. Lam, G. Crum, M. Wirthlin, A. Wilson and A. Stoddard, CHREC space processor: a multifaceted hybrid architecture for space computing, Proc. of the AIAAIUSU Conference on Small Satellites, 2014.

[10] Sterpone, Hagemayer, "A novel fault tolersant and runtime reconfigurable platform for satellite payload processing", IEEE transaction on computers, 2013
[11] J. Khalifat, A. Ebrahim, A. Adetomi and T. Arslan, "A dynamic partial reconfiguration design for camera systems," 2015 NASA/ESA Conference on Adaptive Hardware and Systems (AHS), Montreal, QC, 2015, pp. 17.

[12] F. Nicodemos, O. Saotome and G. Lima, "RTEMS Core Analysis for Space Applications," 2013 III Brazilian Symposium on Computing Systems Engineering, Niteroi, 2013, pp. 125130.

[13] On Line Applications Research Corporation, Huntsville, AL. USA RTEMS Applications C User's Guide, 4.10.99 edition, Jul. 2015.

[14] On Line Applications Research Corporation, Huntsville, AL. USA RTEMS POSIX API User's Guide, 4.10.99 edition, Jul. 2015.

[15] Xilinx , 7 Series FPGAs Configuration User Guide, 2016, (v1.11)

[16] B. Ranft and C. Stiller, "The Role of Machine Vision for Intelligent Vehicles," in IEEE Transactions on Intelligent Vehicles, vol. 1, no. 1, pp. 8 19, March 2016

[17] F. Mühlbauer, M. Großhans and C. Bobda, "Rapid prototyping of OpenCV image processing applications using ASP," 2011 22nd IEEE International Symposium on Rapid System Prototyping, Karlsruhe, 2011, pp. 1622.

[18] A. Cortes, I. Velez and A. Irizar, "High level synthesis using Vivado HLS for Zynq SoC: Image processing case studies," 2016 Conference on Design of Circuits and Integrated Systems (DCIS), Granada, 2016, pp. 16.

[19] Xilinx, Vivado Design Suite User Guide - Partial Recofiguration, 2016, UG909 (v2016.3)

[20] Xilinx, Vivado Design Suite Tutorial - Hierarchical Design, 2016, UG946 (v2016.3)

[21] B. Sellers, J. Heiner, M. Wirthlin and J. Kalb, "Bitstream compression through frame removal and partial reconfiguration," 2009 International Conference on Field Programmable Logic and Applications, Prague, 2009, pp. 476480.

[22] Xilinx, Soft Error Mitigation Controller v4.1 - LogiCORE IP Product Guide, 2017, PG036. 